\title{
Status of Menstrual Dignity during the COVID-19 Pandemics
}

\author{
Anupa Regmi, Devaraj Acharya, Radha Paudel, Samiksha Koirala and Thomas Wenzel* \\ WPA Scientific Section on Primary Health Care, Austria \\ ${ }^{\star}$ Corresponding author: Thomas Wenzel, WPA Scientific Section on Primary Health Care, Austria
}

Received: August 31, 2021; Accepted: September 06, 2021; Published: September 15, 2021

\begin{abstract}
Background: Violence against women takes different forms, often reflecting cultural patterns. Forced segregation and other dangerous or at least discriminatory practices during menstruation can be observed in a number of cultures, such as Nepal, but also in other regions. The present pandemic with its special risks and lockdown measures must be expected to potentially cause additional problems for women in the critical time of menstruation.

Aims and methodology: The aim of our study was to collect information on the experience of women in different regions and identify risk factors for such practices, such as education, health belief systems and bias in the communities together with the impact of the COVID pandemic on these factors. The survey was conducted online to keep safety protocols necessary during the SARS 2 pandemic. To identify possible key factors we conducted a qualitative/mixed method survey resulting in categories and vivid descriptions relating to violence and discrimination. 139 participants, (age range 13-48 years, 85.8 percent female, else LGBT) from different countries, including both low-economy countries with high rates of reported discrimination such as Nepal and India, but also from the US participated in the survey.
\end{abstract}

Results: Patients reported experience of bias and insufficient or incorrect information by parents, and later in the communities. Lockdown measures impacted in some cases, but in general to a lesser degree on access to dignified hygienic measures required during menstruation, as compared to before the pandemics, but was reported to increase the social stress and reduce social support. Shame, insecurity and distress during menstruation continued

and were described as main adverse factors influencing well-being and psychological health.

Keywords: Women, Gender, Menstruation, Discrimination, Dignity, Pandemics, COVID-19

\section{Background}

The World Health Organization (WHO) confirmed the coronavirus outbreak as a pandemic on 11 March 2020. From the outset of the pandemic, the United Nations and various countries are working towards a large-scale, coordinated, and comprehensive health response. Women are disproportionately affected by the COVID-19 crisis because of the gender and social norms combined with the disruption of services and the special challenges of factors such as menstruation or pregnancy [1]. The impact must be seen as multifactorial, including the impact of infection, long COVID, vaccination, and the different COVID prevention and lock-down measures. They have been documented to affect some population groups such as women and migrants in different ways [1-3].

A number of publications have also reported the impact on the physical health of women such as sex hormones, fertility and spontaneous abortion [4-6] and on mental health [7]. Several authors, such as Abuhammad et al. have further observed increased problems related to domestic violence in the Pandemics $[8,9]$. Abdelbadee et al. have further drawn attention to the specific problems to be observed in low-income countries [10].
The pandemic has been exacerbating existing inequalities between women and men in almost all areas of life [3]. However, menstrual discrimination (taboos, stigma, abuses, restrictions, discriminations) has not been studied in the context of the pandemic at least in Asia in spite of the well published earlier concerns in regard to the politics of menstruation during the pandemic by Jahan [11], by the first data published by Aolymat on women in Jordan [12] and several other authors [13-15].

Therefore, this study was conducted to fill this gap mainly by contributing to a better understanding of menstrual dignity and the experiences of women and LGBTQI during menstruation and observed changes during the COVID-19 pandemic.

\section{Methodology}

The study adopted a mixed-method qualitative method integrating qualitative components based on open questions with a short structured questionnaire to elicit general social data and basic information on menstruation related health literacy developed for the study. We also decided to use a multi/transcultural approach by recruiting participants from different countries and cultures as culture has been identified as a major factor in menstrual practices and related 
health belief systems [16]. The survey modules separated questions regarding the situation in general and before the pandemic and those regarding the changes observed during or due to the pandemics.

A survey of 139 participants from different countries was conducted using an open online platform developed for the project.

Not all respondents filled out all items in the survey, though all participants answered to at least $90 \%$ of the questions. The respondents had been recruited through a public platform delivering the survey. All participants gave informed consent during registration, data were stored anonymised on a safe server and will be erased in due time following common data safety protocols.

The age range of participants was between 13-48 years, 85.8 percent were female and 11.2 percent and the rest identified themselves as LGBTQI (Lesbian, Gay, BiSexual, TranSex, Queer and Intersex). The participants were from ten countries: Bangladesh, Bhutan, India, Israel, Madagascar, Namibia, Nepal, Philippines, Uganda, and USA. All respondents had education backgrounds ranging from higher education to master's degree; 22.1 percent finished high school; 58.8 percent held bachelor degrees and 19.1 percent master's degree as highest level of education. Only nine percent of the respondents revealed that they were people with physical or mental disabilities at the time of responding to the online survey.

\section{Findings}

\section{General Findings}

\section{Knowledge about Menstruation}

The respondents reported to have learned about menstruation from different sources, which included mainly their mothers, other family members, school, internet, or from friends. This happened in some participants when they were between age 5-13 years, and in the majority only when they were 10-12 years old.

In the qualitative part of the survey, using the open questions based on an interview guideline, respondents reported:

'My grandmother gave me a "menstruation talk" starting at age eight up till age 13 and gave me information that was appropriate at each age as I come from a family that has a high risk of menstrual problems. Post age 13, I have received informed from friends \& gynecologists'.

'Although school syllabuses contain reproductive and sexual education, students are not properly provided with knowledge. I learnt most of it from my mother and later by studying myself'.

'When my mother was menstruating, my father asked for help from me while cooking.'

\section{Initial Reaction}

Respondents stated that their first reactions after having the menstruation were as follows. Sixty-six percent of respondents felt scared, seven percent felt sick, and seven percent considered menstruation normal and reacted accordingly. Ten percent of responses on this question noted that the question did not apply to them (all members of the LGBTQI group), and another 10 percent had an ambivalent experience.

'When I looked into my pants, I was devastated, thinking I was about to die. Nobody likes looking at blood, in whatever sense'.

'Did I eat something wrong, or did I had an accident that makes my internal parts bleed'

'Damn, will I survive with this massive loss of blood?

'During menarche, I thought I was exposed to some deadly disease and angrily went to my mother and claimed that I feel I would die soon as I am bleeding. Then, my mother explained that you are menstruating and it's a natural process which a woman has to undergo every month.

\section{Discrimination during Menstruation}

Even in regular menstruation, many of the 77 respondents on this items experienced discrimination during menstruation directly and indirectly, as described in the open responses. Mostly, respondents described discrimination as verbal, emotional, as denial of menstrual products, and as impaired mobility. Out of 77 respondents, 23 participants stated that they remained silent while experiencing discrimination. They felt hurt, traumatized and pained though they did not do anything inadequate in their own perception. This group simply tried to ignore discrimination and most of them stated they had no energy to "fight back". Some of them also said that they felt like "avoiding people", "running away" or it her acts of social withdrawal.

'I would not want to be a part of the community where people do not understand and respect a natural phenomenon as such.

'I have left an office internship because my supervisor wasn't sensitive enough. So instead of working from the office, I chose to work in a different department in the same organization. I have also complained about this insensitive behaviour of the supervisor'.

'I feel inferior to the persons who perpetuate violence towards me, and I feel pressured by societal expectations to abide by the greater publics expectations, so therefore I do not feel empowered to speak up even though I am aware I should do so.'

Out of 77, 49 respondents tried fighting back. They educated and empowered themselves by sharing facts and then started raising questions about the experienced acts of discrimination. Some reported to have argued with community and family members, and to have used different forms of direct confrontation, including screaming. Further they reported engaging in teaching about natural phenomena and human rights in the community, and involving community members in a dialogue on menstruation.

'I tried understanding why this is happening and why was it considered a taboo. I usually have these conversations with my mother because she is usually the one who perpetuated the discrimination and had herself not experienced any problems with my family. Still, while visiting the village, I hide the fact that I'm menstruating.'

'When it comes to PMS comments and menstrual stigma from peers, I educate them politely. However, for elders, I have given up on explaining because they won't budge'. 
However, even while fighting back, they experienced feeling tortured, weak, sad, annoyed, oppressed, helpless, depressed, embarrassed, frustrated, worthless, tired, ashamed, guilty, disappointed, and awful (using the verbs in the qualitative part of the online review). Only few positive emotions were reported, such as feeling proud, or courageous.

'Once, while I was visiting Lonavala, there was a temple I wanted to see, and I couldn't because I was during the period. So I asked my mom, "Why can't I go inside the temple just coz I'm bleeding" she told me, "you shouldn't question faith"!!! And I felt very bad. I wanted to see the temple from the inside. But, I was so damn eager to see it, and I couldn't.

'Very bad. Life on this planet exists due to the occurrence of menstruation, and they say we are weak for having it. Really!'

'I feel disappointed at our education system and anger towards those conditioned to perpetuate discrimination by patriarchal mind sets'

'I would surely feel shame, that's awful, but it's always the first emotion I feel when someone perpetuates some sort of violence on me. But then, I'll call back and feel courageous. Then, last, I'll be sad about how people can be so rude and uneducated'

\section{Openness Regarding Menstruation, Disclosure}

Only 29 respondents stated that they freely talk with family members, in most cases because they are health workers (menstruator or non-menstruators) and asked for medicine or pads without any hesitation. The rest of the respondents (n-59) talked only with female members of the family such as mother, elder sister, grandmothers, and aunts and their discussions were more focused on 'do's and 'do not' including restrictions associated with menstruation, menstrual products, and menstrual symptoms or illness.

'Yes, I talk about menstruation with my family. Talks would be something about how to maintain good hygiene during periods and techniques to ease cramps.'

'With my mother only. Not my sister or father. It is very awkward. I only ever ask her for tampons when I run out. I spoke to her when I was younger about what age she got her first period and how to use tampons and pads.'

'As my father does not menstruate, and we were taught not to speak of "these things" with anyone other than mother, my conversation is mostly limited with her'.

80 respondents remained silent in public about menstruation, thinking for example that "it was disrespectful towards elder members of the family". They stated they did not receive support from the family and considered it a matter of "hush and girl thing" (personal or private). Reasons mentioned included "Men should not know anything", "conservative family", "they hate the topic", "would be avoided by men".

"Maybe in African cultures, it is regarded as a taboo. However, we are fighting for fundamental rights, and everyone could be a part of this".
"Because my parents believe menstruation should be kept a secret and that it is not conversation meant for the living room".

However, respondents knew that their friends had been menstruating through observation of their various behaviors, which included blood stained clothes, their experiences of severe cramps, their being more emotional than usual, being hesitant to walk or to walk with precaution, the increased frequency of toilet breaks, avoidance of regular activities, from whispering among girls friends, asking for a favour like a pad, and by their having "pimples". Segregation was common in their experience.

'In Islam, a person is unable to perform "ablution" because it needs proper hygiene, so the person cannot perform religious duties'

'In our culture, girls stay away and are considered untouchable. They are restricted from staying anywhere and from touching anything casually. They shouldn't touch anybody. In the case of males, they shouldn't even go nearby?'

'Yaa. In our society, they stay separately during periods and wear old clothes. So it can be known easily'.

\section{Anxiety during Menstruation in the Bathroom}

Only 23 responses registered that they worried about using the toilets at home due to fear of staining the blood, lack of water, fear of getting scolded, and running out of the menstrual hygienic aids. Respondents reported that they "avoided anybody who knows about the state of menstruating", its being "like a murder scene". Some reported also fears including "fear of contamination from the toilet", "the sound of the opening of the pad", and "fear of some household members".

'As you can touch nothing, you should beg for being handed everything. If you use your bathroom, it should be cleansed.'

'Fear of getting scolded if the floor gets blood on it, lack of water supply-inability to flush the blood out, fear of getting scolded for forgetting used menstrual products wrapped in the bathroom after taking a shower.'

\section{Understanding of Dignified Menstruation}

A total of 62 percent of respondents mentioned that they would perceive freedom during menstruation as dignified menstruation. They considered that hygienic products (access to menstrual pads at home and workplace), freedom of individual behaviour, and family support as being the elements to make menstruation dignified.

\section{Menstruation during the Pandemic}

Out of 104 responses, fifty participants expressed that they experienced increased direct and indirect discrimination as compared to before the pandemic. They expressed varieties of violence in this context: physical (once), verbal, emotional, and denial of services. They reported not being allowed to go to the temple, not allowed to cook food, not allowed to consort with their husbands, prohibition to touch some plants (Tulasi), etc.

In the part of the questionnaire asking for experiences first observed during the pandemics, they reported: 
'"seems like you're ALWAYS on your period," "stop using it as an excuse," "don't you dare bleed on my bed," not much.'

"One of my friends was beaten because she touched a water purifier."

'My brother couldn't really understand when I first menstruated. People wouldn't talk about this subject. He even complained about the smell of the blood. He would also force me to do my chores, and I really felt bad because he found it was unfair that I would go to sleep because of some "random illness once a month."

Respondents experienced difficulties due to travel restrictions to purchase medicine for pain management and menstrual products, as online orders popular in high-income countries during the pandemics are not always available or affordable. On the other hand, when for example the government in India announced a COVID relief package, the menstrual pads were not considered essential by the authorities.

Still, only few of the participants $(n=10)$ reported that they run out of menstrual products, experienced scarcities, or experienced increased expenses, especially after the second and third months of the lockdown . Several participants reported that they were not aware that the frontline health (pathologists, nurse, doctors etc.) workers supporting menstruation practices struggled so much during menstruation due to lack of extra PPE (Personal Protection Equipment's), menstrual products, and hygiene facilities at their workplace, while others commented on the situation especially in India, as mentioned before. Even the media highlighted the scarcity of toilet paper but did not care about menstrual products to cover in their media, as did some governments in their support emergency plans described above.

'When the Indian government was making a list of industries that need to operate during the lockdown to provide necessary items, menstrual products were not included in the initial stage..."

Such restrictions during menstruation were accented during COVID-19 [11], and the Pandemic can be seen as discrimination and inequality in health care as in the above example. Women cannot speak about the discomforts and problems openly, cannot move freely, have to work with discomfort and pain, have difficulties in getting a shower and wash their belongings, feel ashamed when buying pads, feel pressure all the time even when they are at home with family, and expressed that they felt isolated or were marginalised even before the pandemic.

'Girls are treated like as male COVID-19 patients (without special consideration)'

'I always have, pandemic or no, been flabbergasted by the rules of not entering temples during periods, a duality of our society where you are fascinated by the femininity of goddesses but are filled with stigma when it comes to menstruation.

'Women should not talk about it to anyone, especially boys, because it makes you lose dignity. To "control the flow of my blood" and not to make a mess on my bed'.

'We have to work very hard though we are agonizing in pain while the men of the house just sit and relax with no cramps or anything.
'Not able to use the kitchen. I need to ask for someone else to provide something like hot water. The situation is the same during the pandemic as before'.

'This time, I had gone to my husband's home in lockdown. I was asked not to enter the kitchen for four days and avoided drinking milk tea too. I have to use all utensils separately. I feel so bad, but I follow it because I was tired of cooking every morning to evening for about four months. Otherwise, I wouldn't tell anybody. But secretly, I entered the kitchen to get water and food.'

Many menstruating women with a disability, transmen, immigrants, and refugees had suffered more discrimination during menstruation. They were compelled to use old clothes due to the lack of availability of menstrual products. However, only few reported feeling ashamed in managing menstrual products in front of the men's family members, including children.

'During the COVID buying PAD was the main issue trans-man. However, they can't buy the PAD at the time of menstruation because shop owners ask many more questions and they can't answer, so they use a single PAD for up to three days too'.

Only few of the members of this group thought that the menstruating persons were affected more by the pandemic due to their bleeding status.

\section{Earlier Existing Practices of Segregation can be Accentuated}

'My uncles and aunts in the village think that menstruating women are more likely to develop COVID 19, so they isolate them for 14 days when menstruating. However, it's not a very logical conclusion.'

In addition to these practices, some respondents also reported increased difficulties like menorrhagia, uneasiness, or the need to work more due to the absence of domestic help during the pandemic. Similarly, participants reported that they could not buy pain killers to ease the cramps, and their period to be irregular due to lack of exercise or change in lifestyle in this situation. Likewise, some participants used the pad sparingly to minimize the waste and to better hide from non-menstruating sisters and other family members,

'Have to work more at home as domestic helpers are not available because of COVID-19 so I have no rest and have to work through pain'.

'Since I am not at school, during my periods, I am just in bed all day crying until the pain passes. Because I can't go out to buy painkillers, as they are more expensive than ever, and I consequently am in dire need to distract myself from the pain and focus on studying or something. Sometimes, I exercised heavily to dull the pain'.

Other women reported "I used few products to avoid the waste in our bins. I am worried that my non-menstruating sister or father will see the products in the bin and be embarrassed.'

\section{Use and Management of Menstrual Products during the COVID-19 Pandemic}

Regarding use of menstrual products, from the 106 participants who responded to this part of the survey, 82 participants used menstrual 
pads. Three used menstrual cups, and one used tampons. Respondents considered themselves privileged more than others in many ways; such as "living in urban areas", by an "online supply", and because of "access to money". Participants bought menstrual pads from the grocery shop, pharmacy, and online, as they were open even during the lockdown. Few of them purchased in bulk before the imposing of lockdown. Therefore, most respondents, but depending on the local situation, continued the use of menstrual products as before.

'All pharmacies are open since the number of COVID-19 cases is not very high in my area.'

'I always bought a big pack of sanitary pads that is sufficient for 34 months, sometimes more ... so I didn't feel any shortening.'

'I'm fortunate enough to have easy access to products. Medicine shops are open. They are available there'.

'I use a menstrual cup. It's pretty hygienic and reusable, plus it's so comfortable I almost forget that I'm on my period'.

'I had to "stock-pile" tampons before the lockdown, as we are not going to the shops often enough. I am managing okay, but we would be in a difficult situation if we hadn't had money to buy extra tampons.

A total of ten respondents applied alternatives to manage the menstrual blood during lockdown like sleeping, or cloth pads because of financial limitations as the work and salary of their parents were interrupted.

'It's been quite challenging mainly to the girls in my community, lack of money to buy sanitary pads since also parents are no longer working.

The transmen suffered much when they had to get out and buy the pads.

'It's pretty tough because it's not like I can run to shops when I'm out of pads'

Regarding waste management of such menstrual products, 75 respondents said that they were practicing as usual by throwing them in a in garbage tank either at home or in the municipality, seven burned, five re-used the products (cups and cloth), and one buried them in their field. Thus, they did not have significant problems managing the waste due to COVID-19. However, they struggled to wrap them up in newspapers, plastics, by hiding them underneath of bed, etc. due to already pre-pandemic taboo and stigma around menstruation.

'I wrap them in paper and throw them in the dustbin, which is collected by the municipality vehicle garbage.'

'Hardest thing ever. I keep collecting used pads for 2-3 days under my bed. Then, later, when there's no male member around, I cover myself and throw it in the bin downstairs.'

25 (total 76 responses) respondents reported hiding their menstrual pads and increased increased problems during the pandemic. They experienced irritation, sadness due to the unavailability of the dustbin, and a few experienced foul odors, unhygienic conditions and felt shy in front of others.

One respondent described her experience "I don't like the smell of the product and my blood. And I don't want my family to see and smell of that disposed stuff. I don't want my family to see something that was near my private area. And I am also worried that the adhesives might give up, and the folded pad opens up, revealing a "bad" scene".

\section{Discussion}

Pandemics or any other disaster can have a severe physical impact on menstruation and the health of women [17-20]. Menstruating women are experiencing increased discrimination during the COVID-19 pandemic due to silence and ignorance about menstruation, but also in logistics and access to menstruation pads or cups during the pandemics as observed by our group. This can result in difficult situations for both the women and for their health care workers, as reflected in extreme measures - as example frontline health workers reportedly used birth control pills to stop menstruation at Wuhan, China. However, women have a dire need for menstrual dignity through more freedom during menstruation. The impact of menstrual discrimination has a multi-level impact that affects mental, physical and social health and might lead to violation of the human rights of the women. The needs and priorities of women should be scrutinized and need to be addressed even in shifting priorities of service providers during quarantine, isolations, hospitals, travel restrictions and curfews, the ongoing financial crisis. Scarcity of menstrual products and other essentials in some countries might be affected to a different degree. Communities and families need education and improved health literacy to fight health belief models leading to discrimination or violent acts such as forced segregation.

The observations in our mixed method study are limited by the small sample size and by the methodology based on an online survey and can therefore not provide representative data on the countries of the participants, that can be generalized. Still, the results identify and highlight some of the problems pre-existing and those accentuated or created during the pandemic.

\section{Conclusion and Recommendations}

Menstrual discrimination prevails in many countries and the problem was neglected across all the countries during the COVID-19 pandemic in our study. Since menstrual discrimination is a form of gender-based violence, it is continuously manifested in various forms. Based on the findings, our recommendations are as following:

1. Information should be available everywhere in numerous languages on the physiology of menstruation and all aspects of menstrual dignity to increase health literacy for all genders and decrease stigma and discrimination based on inadequate knowledge and traditional cultural practices or belief systems.

2. The menstrual products should be included in the COVID-19 response packages, and other humanitarian response materials.

3. The provision of water, menstruation-friendly toilet/bathroom, hand sanitizers, and a mechanism for safe, discrete and low barrier waste management needs to be ensured in all settings, including temporary shelters, as explored by some authors [21].

4. Programs as described in 1 , focusing on menstrual dignity need to be continued across all programs as a cross-cutting concern. 


\section{References}

1. Abate BB, Kassie AM, Kassaw MW, Aragie TG, Masresha SA (2020) Sex difference in coronavirus disease (COVID-19): a systematic review and meta-analysis. BMJ Open 10: e040129. [crossref]

2. Türkan Akkaya-Kalayci ODK, Thomas Wenzel, Anthony Chen VC, Zeliha ÖzlüErkilic (2020) The impact of the COVID-19 pandemic on mental health and psychological well-being of young people living in Austria and Turkey: a multicenter study International Journal of Environmental Research and Public Health 17: 9111. [crossref]

3. Abufaraj M, Eyadat Z, Al-Sabbagh MQ, Nimer A, Moonesar IA, et al. (2021) Genderbased disparities on health indices during COVID-19 crisis: a nationwide crosssectional study in Jordan. Int J Equity Health 20: 91.

4. Cosma S, Carosso AR, Cusato J, Borella F, Carosso M, et al. (2021) Coronavirus disease 2019 and first-trimester spontaneous abortion: a case-control study of 225 pregnant patients. Am J Obstet Gynecol 224: 391 e1-e7. [crossref]

5. Xiong Z, Li P, Lyu H, Luo J (2021) Observational Study of Working from Home during the COVID-19 Pandemic Using Social Media Data. JMIR Med Inform.

6. Bagli I, Ocal E, Yavuz M, Uzundere O, Bozkurt F (2021) Maternal deaths due to COVID-19 disease: The cases in a single center pandemic hospital in the south east of Turkey. J Obstet Gynaecol Res.

7. Ahorsu DK, Imani V, Lin CY, Timpka T, Brostrom A, et al. (2020) Associations Between Fear of COVID-19, Mental Health, and Preventive Behaviours Across Pregnant Women and Husbands: An Actor-Partner Interdependence Modelling. Int J Ment Health Addict 1-15. [crossref]

8. Abuhammad S (2021) Violence against Jordanian Women during COVID-19 Outbreak. Int J Clin Pract 75: e13824. [crossref]

9. Abujilban S, Mrayan L, Hamaideh S, Obeisat S, Damra J (2021) Intimate Partner Violence Against Pregnant Jordanian Women at the Time of COVID-19 Pandemic's Quarantine. J Interpers Violence 886260520984259. [crossref]

10. Abdelbadee AY, Abbas AM (2020) Impact of COVID-19 on reproductive health and maternity services in low resource countries. Eur J Contracept Reprod Health Care 25: 402-404. [crossref]
11. Jahan N (2020) Bleeding during the pandemic: the politics of menstruation. Sex Reprod Health Matters 28: 1801001. [crossref]

12. Aolymat I (2020) A Cross-Sectional Study of the Impact of COVID-19 on Domestic Violence, Menstruation, Genital Tract Health, and Contraception Use among Women in Jordan. Am J Trop Med Hyg 104: 519-525. [crossref]

13. Wilbur J, Kayastha S, Mahon T, Torondel B, Hameed S, et al. (2021) Qualitative study exploring the barriers to menstrual hygiene management faced by adolescents and young people with a disability, and their carers in the Kavrepalanchok district, Nepal. BMC Public Health 21: 476.

14. Thapa S, Aro AR (2021) 'Menstruation means impurity': multilevel interventions are needed to break the menstrual taboo in Nepal. BMC Womens Health 21: 84. [crossref]

15. Levitt RB, Barnack-Tavlaris JL (2020) Addressing Menstruation in the Workplace: The Menstrual Leave Debate. In: Bobel C, Winkler IT, Fahs B, Hasson KA, Kissling EA, Roberts TA, editors. The Palgrave Handbook of Critical Menstruation Studies. Singapore 561-575. [crossref]

16. Paudel Radha A, Mili, Kletecka-Pulker, Maria, Wenzel, Thomas (2019) The Construction of Power in Nepal: Menstrual Restriction and Rape Arch Women Health Care 2.

17. Li F, Lu H, Zhang Q, Li X, Wang T, Liu Q, et al. (2021) Impact of COVID-19 on female fertility: a systematic review and meta-analysis protocol. BMJ Open 11: e045524.

18. Li K, Chen G, Hou H, Liao Q, Chen J, Bai H, et al. (2021) Analysis of sex hormones and menstruation in COVID-19 women of child-bearing age. Reprod Biomed Online 42: 260-267. [crossref]

19. Mishra N, Sharma R, Mishra P, Singh M, Seth S, Deori T, et al. (2020) COVID-19 and Menstrual Status: Is Menopause an Independent Risk Factor for SARS Cov-2? J Midlife Health 11: 240-249. [crossref]

20. Phelan N, Behan LA, Owens L (2021) The Impact of the COVID-19 Pandemic on Women's Reproductive Health. Front Endocrinol (Lausanne) 12: 642755. [crossref]

21. Hirai M, Nyamandi V, Siachema C, Shirihuru N, Dhoba L, et al. (2021) Using the Water and Sanitation for Health Facility Improvement Tool (WASH FIT) in Zimbabwe: A Cross-Sectional Study of Water, Sanitation and Hygiene Services in 50 COVID-19 Isolation Facilities. Int J Environ Res Public Health 18: 5641. [crossref]

\section{Citation:}

Regmi A, Acharya D, Paudel R, Koirala S, Wenzel T (2021) Status of Menstrual Dignity during the COVID-19 Pandemics. ARCH Women Health Care Volume 4(4): 1-6. 